\title{
Mit Heilerde natürlich behandeln - innerlich und äußerlich
}

Ein traditionsreiches Naturheilmittel im Fokus der Wissenschaft

Bernhard Uehleke

Heilerde besitzt aufgrund ihrer geologischen Zusammensetzung und Entstehungsweise einen hohen Feinheitsgrad und damit eine große Oberfläche. Deshalb hat sie ein besonders ausgeprägtes Bindungsvermögen für Bakterien, Säuren und andere Stoffwechselprodukte. Sie eignet sich dadurch für die innere Anwendung bei gastroenterologischen Störungen sowie äußerlich bei Hauterkrankungen.

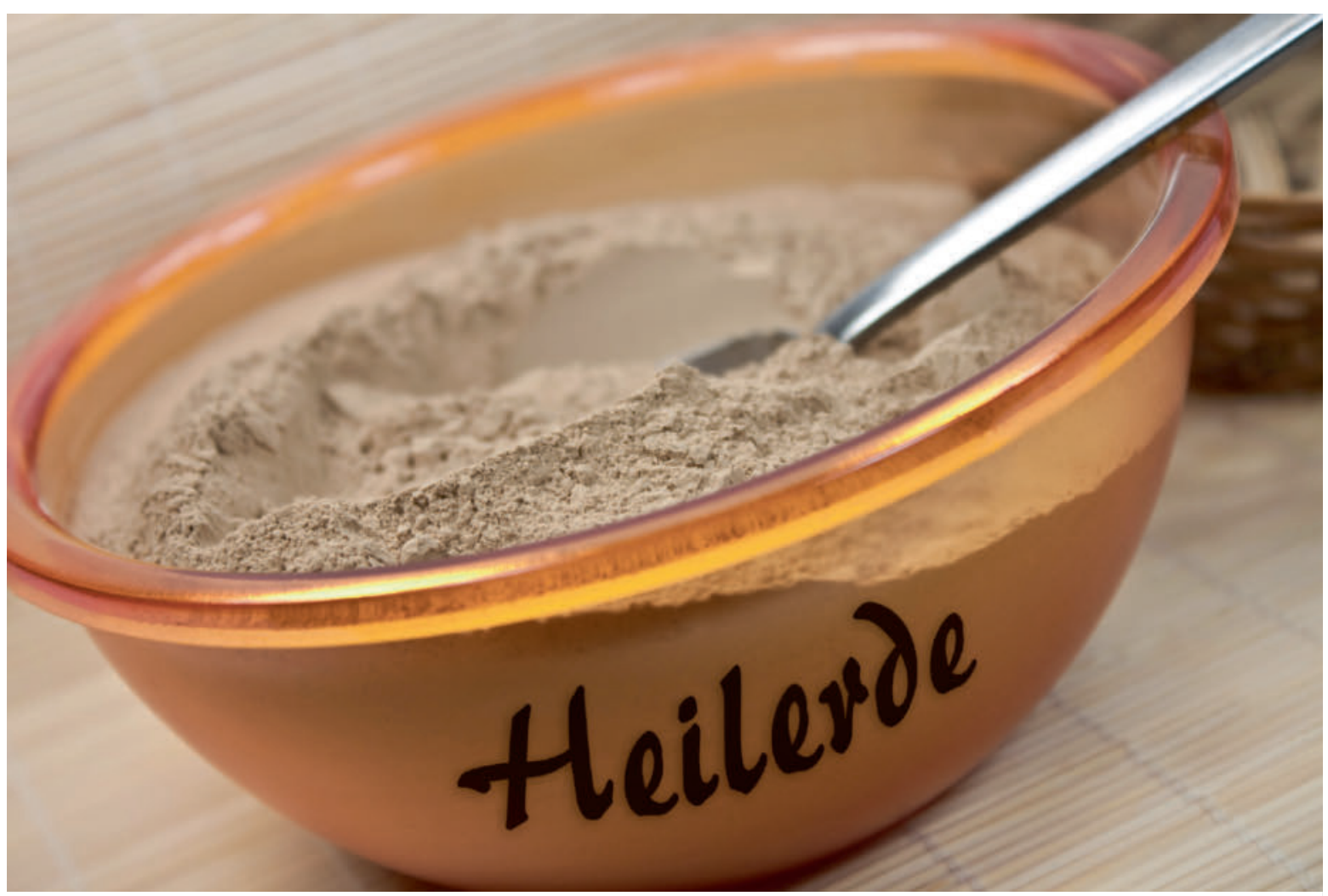

Die braune Heilerde eignet sich als Pulver für die innerliche Anwendung bei zahlreichen Erkrankungen und äußerlich für die Hautund Wundheilung. Foto: PhotoSG/Adobe Stock 


\section{Geschichtliches}

Verschiedene Heilerden spielten bereits in der Antike eine große Rolle, sowohl für die innerliche als auch für die äußerliche Anwendung. Sie wurden über große Entfernungen gehandelt und waren insbesondere in Notzeiten sehr wertvoll - mitunter wurde Heilerde mit Gold aufgewogen.

Der Naturheilkundler Adolf Just (1859-1936) errichtete 1896, inspiriert u. a. von Sebastian Kneipp, seine Naturheilanstalt „Jungborn“ im Harz. Neben „Lichtluftbaden“ und Sonnenbaden wandten die Patienten dort Erdumschläge an und nahmen Heilerde ein. 1908 übertrug Adolf Just die Leitung des „Jungborn“ seinem jüngeren Bruder Rudolf (1877-1948). Seit 1918 vertreibt die Familie eine spezielle feine Lösserde unter dem Namen Luvos-Heilerde über die „Heilerde-Gesellschaft“. Diese Heilerde wurde eines der bekanntesten und beliebtesten Mittel in der Hausapotheke.

\section{Eigenschaften der Heilerde}

Löss ist aus Gesteinen entstanden, die mit den eiszeitlichen Gletschern der letzten Eiszeit (Diluvium) aus Skandinavien nach Deutschland transportiert und dabei bis zur Pulverform zerkleinert wurden. Dieses Gesteinspulver ist dann durch den Wind an seine jetzige Lagerstätte geweht worden und zu Löss „verwittert“.

Luvos-Heilerde verfügt über eine ideale natürliche Mischung von Mineralien und Spurenelementen. Darunter sind viele lebensnotwendige Mineralien und Spurenelemente, etwa Calcium, Kalium und Magnesium. Heilerde ist ein reines Naturprodukt, das keine chemischen Zusätze wie Duft-, Farb- oder Konservierungsstoffe enthält.

Aufgrund ihrer Zusammensetzung kann Luvos-Heilerde schnell und effektiv überschüssige Säure abpuffern (binden) ( $\triangleright$ Abb. 1). Das Säurebindungsvermögen erreicht den vom BfArM (Bundesinstitut für Arzneimittel und Medizinprodukte) empfohlenen Wert für Antazida von 25 mVal pro Einzeldosis. Andere Heilerden weisen diese Zusammensetzung nicht auf und sind beispielsweise bei Sodbrennen weniger gut geeignet.

Merke

Luvos-Heilerde ist derzeit das einzige Naturarzneimittel mit dem Wirkstoff Heilerde, das in Deutschland gegen Sodbrennen, säurebedingte Magenbeschwerden und Durchfall zugelassen ist.

Durch den hohen Feinheitsgrad weist die Heilerde eine große Oberfläche mit einem besonderen Bindungsvermögen auf. Damit kann sie mit anderen Stoffen in Wechselwirkung treten und diese ad- und absorbieren (anlagern und wie ein Schwamm aufsaugen). Heilerde bindet Bakterien sowie deren schädliche Stoffwechselprodukte und Gifte - ähnlich wie medizinische Kohle oder pflanzliche

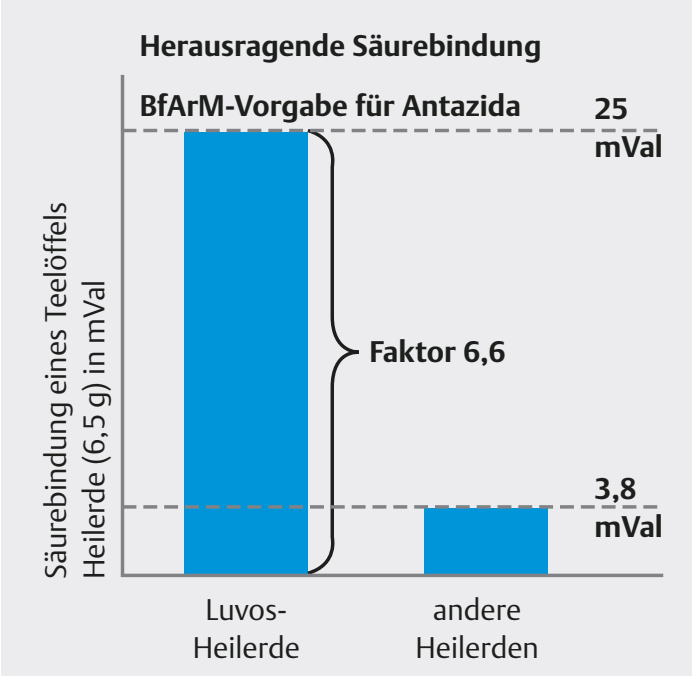

-Abb. 1 Säurebindungskapazität von Luvos-Heilerde im Vergleich zu anderen Heilerden, angegeben in mVal.

Schleimstoffe. Auch Cholesterin und Gallensäuren werden von der Heilerde gebunden.

Entsprechend der gewünschten therapeutischen Wirkung haben die Heilerdeprodukte anwendungsspezifische Feinheitsgrade:

- ultrafein, fein: Sodbrennen, säurebedingte Magenbeschwerden, Durchfall

- magenfein: Reizdarm und Reizmagen

- mikrofein: Bindung von Cholesterin und Fetten aus der Nahrung

- imutox: Entgiftung, zum Schutz vor freien Radikalen, bei Histaminintoleranz

- hautfein: Akne, Haut-, Muskel- und Gelenkbeschwerden, Entzündungen

\section{Wie ist Heilerde einzunehmen?}

Ein bis zwei Messlöffel Heilerde-Pulver oder Portionsbeutel mit Granulat werden zweimal täglich eingenommen. Man rührt das Pulver in Wasser oder Tee ein und trinkt es dann in kleinen Schlucken.

Merke

Heilerde schmeckt weitgehend neutral und nur leicht "erdig“.

Als besonders komfortabel einzunehmende Darreichungsform gibt es Heilerde auch in Kapseln und als Granulat. Das Heilerde-Granulat wird unzerkaut mit reichlich Flüssigkeit heruntergeschluckt. Die Kapseln werden unzerkaut mit etwas Flüssigkeit eingenommen.

Zur Bindung von Cholesterin, Fetten und Schadstoffen aus der Nahrung sollte die Einnahme der Heilerde jeweils zu den Mahlzeiten erfolgen. In den übrigen Fällen wird Heil- 


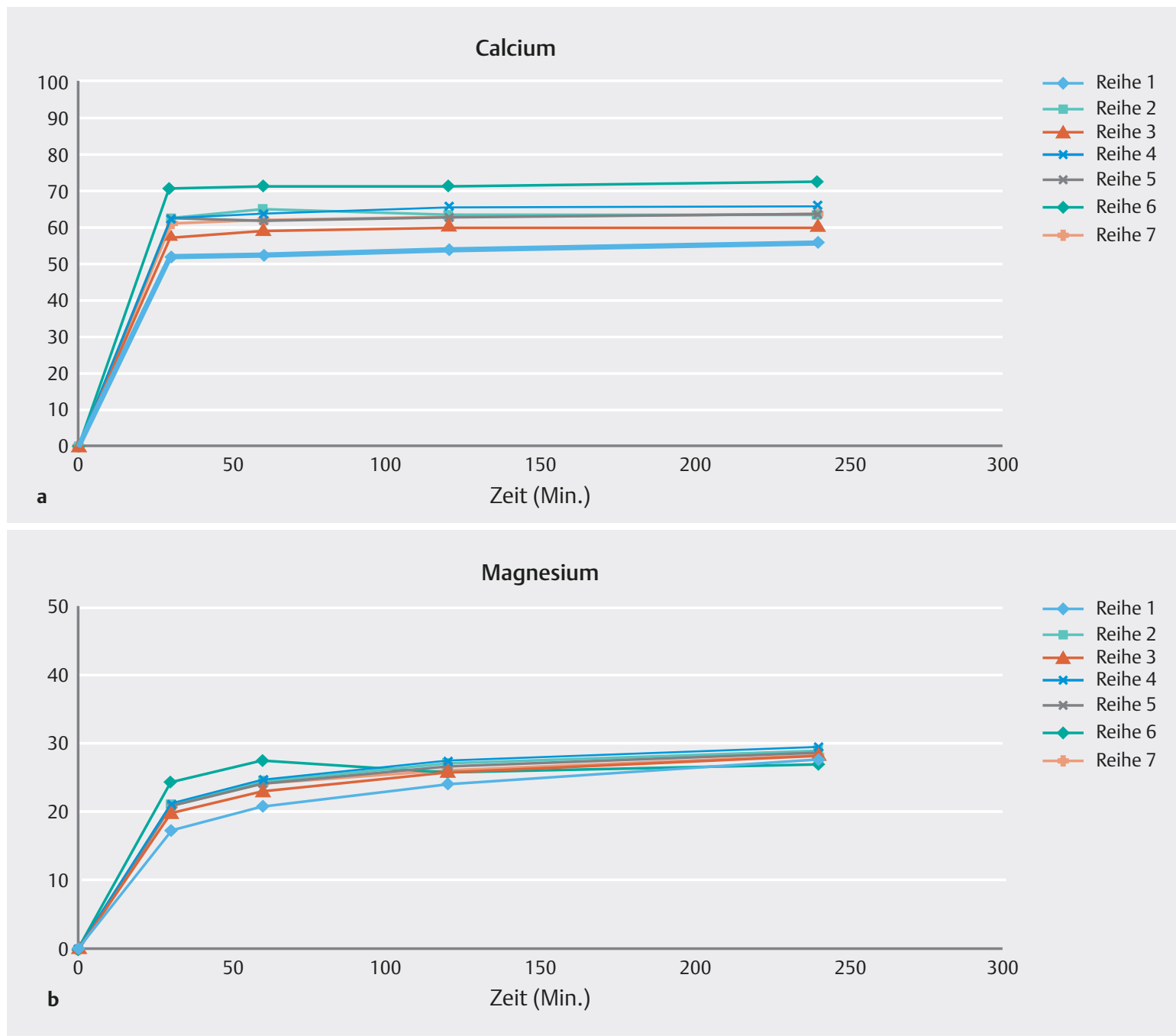

Abb. 2 Bestimmung der mobilisierbaren Inhaltsstoffe von Heilerde in Prozent am Beispiel der Mineralstoffe Calcium und Magnesium [1].

erde morgens nüchtern und abends vor dem Schlafengehen sowie bei Bedarf eine halbe Stunde vor oder nach dem Essen eingenommen, wenn nicht die Beschwerden oder die Einnahme anderer Arzneimittel für eine Einnahme zu anderen Zeitpunkten sprechen.

\section{PRAXISTIPP}

Besonders praktisch, um auch unterwegs immer die richtige Dosis dabei zu haben, sind Portionsbeutel.

\section{Indikationen zur inneren Anwendung}

\section{Natürliche Hilfe bei Durchfall}

Mit Heilerde können akute und chronische Durchfallerkrankungen behandelt werden. Die mineralische Struktur von Luvos-Heilerde bietet eine große innere Oberfläche. So bindet Heilerde im Darm Bakterien und deren schädliche Stoffwechselprodukte. Zusammen mit der Heilerde werden diese dann mit dem Stuhl ausgeschieden, und die gestörte Darmflora findet ohne Nebenwirkungen zu ihrer natürlichen Balance zurück.

Der Körper nimmt Mineralien und Spurenelemente aus der Heilerde auf, was sich positiv auf den Elektrolythaushalt auswirken kann - ein großes Plus gegenüber medizinischer Kohle. Neben Natrium-, Kalium-, Chloridionen usw. werden Calcium- und Magnesiumionen besonders gut herausgelöst ( $>$ Abb. 2). Aus einer Tagesdosis LuvosHeilerde (26g) wird etwa $1 \mathrm{~g}$ Calcium gelöst, sodass der empfohlene Tagesbedarf von 1000 mg Calcium vollständig durch Heilerde gedeckt werden kann.

\section{Natürlich gegen Sodbrennen}

Aus naturheilkundlicher Sicht sollte man Sodbrennen mit einfachen Maßnahmen vorbeugen und die nicht unproblematische langfristige Säureblockade vermeiden. Dies geschieht zum Beispiel, indem man Auslöser wie fettige, süße und scharfe Speisen, Kaffee, Tee, Alkohol, Nikotin und 

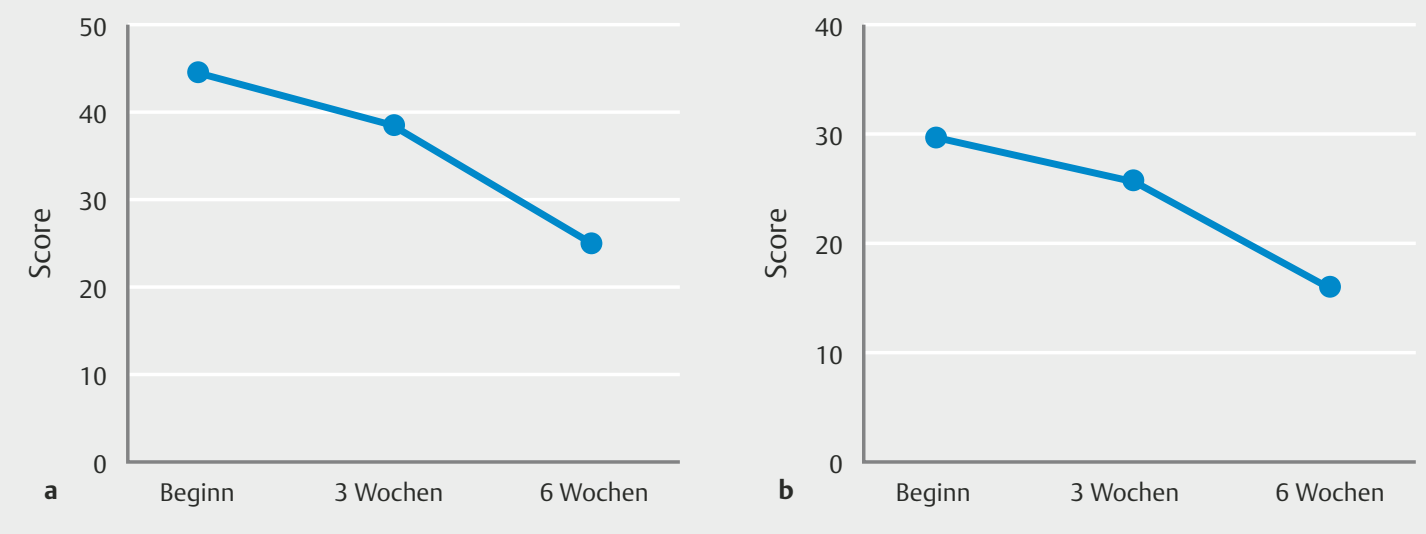

- Abb. 3 Grafische Darstellung der Ergebnisse einer Anwendungsbeobachtung nach 6-wöchiger Behandlung mit Luvos-Heilerde bei Reizmagen (a) und Reizdarm (b). Dargestellt sind die Summenwerte der Gesamtbeschwerden nach Patientenangaben [2].

XXL-Mahlzeiten möglichst meidet, Übergewicht reduziert und auf eine ausreichende Nachtruhe, ggf. mit erhöhtem Oberkörper, achtet.

In einer aktuellen Studie bei Patienten mit non-erosiver Refluxkrankheit zeigte Heilerde ultrafein eine innerhalb von Minuten einsetzende Linderung von akutem Sodbrennen und insgesamt eine deutliche Abnahme der „Anfälle“ sowie aller relevanten Beschwerden im Verlauf über 2 Wochen.

\section{Natürlich gegen Reizdarm und Reizmagen}

Funktionelle Magen-Darm-Beschwerden im oberen Verdauungstrakt, die als sog. dyspeptische Beschwerden bzw. Reizmagen bezeichnet werden, gehen mit Schmerzen, Krämpfen und Missempfindungen im gesamten Bauchbereich einher. Ebenfalls zeigen sich Appetitlosigkeit, vorzeitiges Völlegefühl, Magendruck, verzögerte Magenentleerung oder Beschwerden im Bereich der ableitenden Gallenwege.

Funktionelle Magen-Darm-Beschwerden im unteren Verdauungstrakt, die als Reizdarm bezeichnet werden, gehen neben krampfartigen Schmerzen mit Durchfall, Blähungen oder Verstopfung einher. Für die Beschwerden können keine organischen Ursachen diagnostiziert werden. Andere mögliche Krankheiten wie chronische Dickdarmentzündungen, Darmkrebs, Nahrungsmittelunverträglichkeiten oder Magen-Darm-Infekte müssen ausgeschlossen sein.

Ziel einer Anwendungsbeobachtung an der Abteilung Naturheilkunde der Charité Berlin war es, die Wirksamkeit von Luvos-Heilerde bei Patienten mit funktionellen Magen-Darm-Störungen zu untersuchen [2]. In der Studie wurden insgesamt 64 Patienten mit verschiedenen funktionellen Magen-Darm-Beschwerden untersucht. Männer und Frauen waren im gleichen Verhältnis dabei mit einer weiten Altersverteilung und einem Altersdurchschnitt von
50 Jahren. Ein Großteil der Patienten hatte bereits jahrebzw. jahrzehntelang Beschwerden. $60 \%$ der Patienten nahmen innerhalb der letzten 6 Wochen und über die Beobachtungsperiode von 6 Wochen andere Magen-Darm-Mittel ein.

Da die Beschwerden im Einzelnen völlig unterschiedlich waren, wurde für die globale Aussage zur Wirksamkeit ein Fragebogen verwendet, bei dem einzelnen Symptomen je nach Schweregrad und Häufigkeit bestimmte Punktwerte zugeordnet wurden, die anschließend zusammengezählt wurden. Im Ergebnis zeigte sich, dass die Patienten bei den Gesamtbeschwerden des oberen Verdauungstraktes, ausgehend von einem mittleren Wert von 44, nach 6-wöchiger Behandlung eine Besserung auf rund 25 zeigten. Bei einer Zwischenuntersuchung nach 3 Wochen war die Besserung bei Weitem noch nicht so stark ausgeprägt ( $>$ Abb. $3 a)$.

Ähnlich verhielt sich der Gesamtwert der Beschwerden im unteren Verdauungstrakt (z. B. Reizdarm, Darmkrämpfe, Durchfall, Verstopfung): Hier besserten sich im Mittel die Beschwerden von einem Summenwert von 30 auf 16 nach 6 Wochen Behandlung mit Luvos-Heilerde ( $\triangleright$ Abb. 3b). Da aber in einer solchen Studie auf alle möglichen Nebenwirkungen besonderes Augenmerk gelegt wird, ist es nicht verwunderlich, dass einige Patienten neue und meist vorübergehende Symptome entwickelten.

Als Ergebnis der Studie ist festzuhalten, dass sich funktionelle Beschwerden nach einer längeren Behandlungsdauer mit Luvos-Heilerde deutlich bessern.

\section{Natürlich weniger Cholesterin}

Luvos-Heilerde mikrofein kann Cholesterin und Fette wie ein Schwamm direkt aus der Nahrung binden und transportiert sie über den Verdauungstrakt aus dem Körper. An die Heilerde gebunden kann das Cholesterin nicht mehr in die Blutbahn gelangen, sich an den Gefäßwänden als Plaque anlagern und dort den Prozess der Arterienverkal- 
kung (Arteriosklerose) fördern, die sogar in einen Herzinfarkt münden kann.

\footnotetext{
Merke

Ein zu hoher Cholesterinspiegel zählt neben Rauchen, Bewegungsmangel, Diabetes, Bluthochdruck und Übergewicht zu den wichtigsten Risikofaktoren für Herz-Kreislauf-Erkrankungen.
}

In-vitro-Versuche zeigen, dass 1,5 Messlöffel (ca. $10 \mathrm{~g}$ ) Heilerde mikrofein im Magenmilieu (pH 3,4) 178 mg Cholesterin binden können - dies entspricht etwa dem Cholesteringehalt eines großen Hühnereis [3].

Luvos-Heilerde mikrofein kann außerdem Gallensäuren binden, sodass diese vermehrt ausgeschieden werden ( $\triangleright$ Tab. 1). Die Neubildung von Gallensäuren - sie dienen der Fettverdauung und Fettaufnahme aus der Nahrung - wird auf natürliche Weise angeregt. Da Gallensäuren aus Cholesterin gebildet werden, kann dies zusätzlich zu einem günstigen Einfluss der Heilerde auf den Cholesterinspiegel führen.

\section{Natürlich wirksam zur Entgiftung}

Luvos-Heilerde imutox wurde speziell für die tägliche Entgiftung entwickelt. Sie kann aufgrund ihrer besonderen Bindungseigenschaften die Körperentgiftung, Darmsanierung und Entschlackung unterstützen. Zudem bindet sie belastende Substanzen wie Schwermetalle (z. B. Blei, Cadmium, Cäsium und Quecksilber) ( Tab. 2), Weichmacher (z. B. Phthalate) sowie Bakterien und Schimmelpilzgifte aus der Nahrung und leitet diese zuverlässig aus. Zusätzlich baut Heilerde im Gastrointestinaltrakt als mineralischer Katalysator freie Radikale aus der Nahrung ab. Damit eignet sich Heilerde imutox zur täglichen Entgiftung - empfohlen bei Detox- und Fastenkuren.

Bei Entgiftungs- und Fastenkuren sowie Diäten kann LuvosHeilerde imutox unterstützend angewandt werden. Beim Heilfasten nach Uehleke wird die Belastung der Leber durch Heilerde deutlich reduziert, da gallepflichtige Entgiftungsprodukte der Leber sonst erneut resorbiert werden und erneut die Leber belasten. Insgesamt wird beim Heilfasten nach Uehleke die Verträglichkeit des Heilfastens verbessert.

\section{Bessert Symptome einer Histaminintoleranz}

Sehr hohe Histaminkonzentrationen lösen bei jedem Menschen Vergiftungserscheinungen aus. Patienten mit einer Histaminintoleranz reagieren aber schon auf kleine Mengen mit Beschwerden. Als Ursache für die Intoleranz wird ein gestörter Abbau vermutet, vor allem durch fehlende oder inaktive Diaminoxidase (DAO). Das Enzym kann auch durch Medikamente wie Acetylsalicylsäure und andere Stoffe wie Rotwein gehemmt werden, was die Histaminbelastung zusätzlich erhöht. Die Symptome variieren sehr stark. Häufig treten Kopfschmerzen und Gesichtsrö- 
- Tab. 1 Gallensäurebindung an $10 \mathrm{~g}$ Luvos-Heilerde mikrofein in $100 \mathrm{ml}$ konjugierter Gallensäurelösung bei $37^{\circ} \mathrm{C}$ und pH-Wert 3,4.

\begin{tabular}{l|l|l|l|l|}
$\begin{array}{l}\text { Gallen- } \\
\text { säure }\end{array}$ & $\begin{array}{l}\text { Anfangswert } \\
{[\mathbf{m g} / \mathbf{1 0 0} \mathbf{m l}]}\end{array}$ & \multicolumn{3}{|l|}{ Bindung von Gallensäure [in \%] } \\
\hline & $\begin{array}{l}\text { nach } \\
\mathbf{1} \text { Stunde }\end{array}$ & $\begin{array}{l}\text { nach } \\
\text { 2 Stunden }\end{array}$ & $\begin{array}{l}\text { nach } \\
\mathbf{4} \text { Stunden }\end{array}$ \\
\hline TUDCA & 7,46 & 9,25 & 11,15 & 13,35 \\
\hline TCDCA & 7,69 & 17,64 & 26,27 & 31,80 \\
\hline GUDCA & 6,43 & 3,30 & 5,69 & 9,37 \\
\hline GCDCA & & 19,82 & 26,86 & 30,81 \\
\hline $\begin{array}{l}\text { TUDCA: Tauroursodeoxycholsäure }(150 \mu \text { mol); TCDCA: Taurochenodeo- } \\
\text { xycholsäure (150 } \mu \text { mol); GUDCA: Glycoursodeoxycholsäure }(150 \mu \text { mol); } \\
\text { GCDCA: Glycochenodeoxycholsäure }(100 \mu \text { mol) }\end{array}$
\end{tabular}

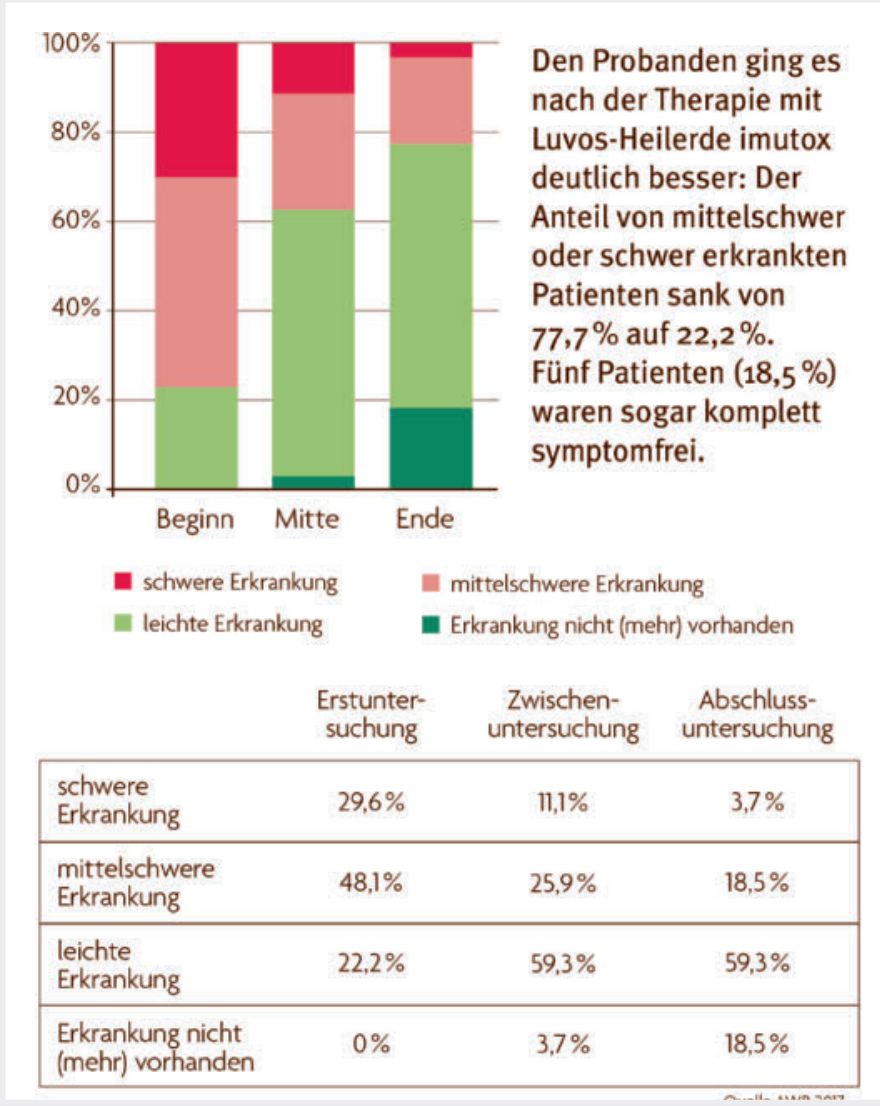

Abb. 4 Ergebnisse einer Anwendungsbeobachtung mit Luvos-Heilerde imutox bei 27 Patienten mit Histaminintoleranz.

te (Flush) sowie Bauchschmerzen und Durchfall auf. Etwas seltener kommt es zur Schwellung der Nasenschleimhaut, Blähungen und Übelkeit oder Herzrasen. Auch Ausschlag, Juckreiz, Müdigkeit, Schwindel und Kreislaufprobleme kommen vor.
- Tab. 2 Adsorption von Schwermetallen und belastenden Substanzen im Magensaftmilieu nach 2 Stunden an LuvosHeilerde.

\begin{tabular}{|l|l|}
\hline Schwermetall & Adsorption \\
\hline Blei & $100,0 \%$ \\
\hline Cäsium & $98,8 \%$ \\
\hline Cadmium & $61,1 \%$ \\
\hline Quecksilber & $99,1 \%$ \\
\hline
\end{tabular}

\section{Anwendungsbeobachtung bei Histaminintoleranz}

Eine Therapie mit Heilerde imutox vermindert die Resorption des Histamins und kann bei dieser Erkrankung lohnend sein - das ergab jetzt eine als Anwendungsbeobachtung (AWB) angelegte Studie: 27 Patienten mit langjährig gesicherter Histaminintoleranz erhielten ergänzend zu ihrer gewohnten, histaminarmen Diät von ihren Ärzten die Heilerde gemäß Gebrauchsinformation zum Einnehmen. Wirksamkeit und Verträglichkeit wurden zu Beginn, nach der halben Therapiezeit und am Ende erhoben - im Schnitt über 26 Tage (Median: 22 Tage).

Die Wirksamkeit der Therapie wurde durch die Behandler am Ende für 23 Patienten (85,1\%) mit „sehr gute Besserung“ bzw. „gute Besserung“ beurteilt. 4 Patienten $(14,8 \%)$ konnten nach rund 3 Wochen Behandlung mit dem Heilerde-Pulver auf die histaminarme Diät verzichten, 5 Patienten (18,5\%) waren sogar symptomfrei (\Abb. 4). Die Zahl der Patienten mit „leichter Erkrankung“ zu Beginn der AWB betrug $n=6(22,2 \%)$ und belief sich am Ende auf $n=16(59,3 \%)$.

\section{Merke \\ Heilerde als Naturheilmittel kann vielen Patienten die Diät ersparen.}

Die Einschätzung der Behandler spiegelt sich auch in den Patientenfragebögen wider. 23 der befragten 27 Patienten $(85,1 \%)$ vergaben für die Wirkung der Heilerde auf die Symptome der Histaminintoleranz eine positive Gesamtbewertung: 48,1\% bewerteten sie als „gut“ (13 Patienten), 33,3\% als „sehr gut“ (9 Patienten) und 3,7\% als „ausgezeichnet“ (1 Patient). Die Lebensqualität der Patienten verbesserte sich deutlich: 77,8 \% fühlten sich nach der Heilerde-Therapie durch ihre Beschwerden kaum oder überhaupt nicht mehr beeinträchtigt vs. 25,9\% zu Beginn.

Bei keinem Patienten wurden schwere unerwünschte Effekte beobachtet. Ein Patient berichtete über 4 Tage eine Verstopfung als unerwünschtes Ereignis, die aber nach Dosisreduktion abklang. 


\section{Äußerliche Behandlung}

Äußerlich wird Heilerde schon seit Jahrtausenden angewendet. So wie der Urmensch wälzen sich auch viele Tiere im Schlamm, um ihr Fell mit einer schützenden Kruste zu bedecken. Eine Erdkruste schützt die Haut besser als jedes Sonnenschutzmittel vor UV-Strahlung, vor Insekten genauso wie vor Brennnesseln oder dornigem Gestrüpp.

Merke

Hildegard von Bingen empfahl zur Behandlung von Verletzungen heilende Erden für die Wundheilung.

Heilerde wird angewendet als Gesichtsmaske, Peeling, Umschlag, Verband und Packung sowie in Spülungen und Bädern oder als Puder bei:

- Hautbeschwerden

- Entzündungen

- Muskel- und Gelenkbeschwerden (Sportverletzungen)

- Haut- und Haarpflege

Luvos-Heilerde hautfein steht dazu als Pulver sowie als gebrauchsfertige Paste zur Verfügung.

\section{Wirkung bei äußerlicher Anwendung}

Luvos-Heilerde wirkt rein physikalisch über ihre große Oberfläche und die bei der Trocknung entstehende Saugwirkung von innen nach außen $(50-150 \mathrm{mmHg}$ Saugdruck). Fett, Talg, Wundsekrete und andere Flüssigkeiten sowie darin gelöste und ungelöste Stoffe und Bakterien werden aufgenommen und gebunden. Die Bildung gesunden Gewebes wird gefördert (wundheilungsfördernd). Je nach Behandlungsziel können wärmende oder kühlende Effekte genutzt werden.

\section{Anwendung bei Hauterkrankungen}

Die äußerliche Anwendung von Heilerde kann besonders empfohlen werden bei Acne vulgaris, Rosacea (Gesichtsrose), Psoriasis (Schuppenflechte), Urtikaria (Juckreiz), Insektenstichen, Brandwunden, Sonnenbrand, Herpes labialis, Herpes zoster und Stomatitis aphthosa.

\section{PRAXISTIPP}

Bei der äußerlichen Anwendung zur Behandlung von Hauterkrankungen mit Heilerde ist unbedingt auf den individuellen Hautzustand Rücksicht zu nehmen. Daher kann die Anwendungshäufigkeit sehr stark variieren - von rund einmal pro Woche bis zu täglich.

Auch bei großflächiger Anwendung fühlt sich die Heilerde sehr angenehm an und blättert nicht so schnell ab, obwohl sie beim Trocknen schrumpft und Risse bildet. Wenn schließlich die getrocknete Heilerde abgerieben oder abgespült wird, ist die Haut so gut von auf der Haut liegenden Stoffen und Erregern gereinigt, dass praktisch kein Fettfilm mehr vorhanden ist; diese Entfettung und Hautreinigung ist genauso intensiv wie mit chemischen Lösungsmitteln (Alkohol), aber weitaus schonender!

\section{Akne, Pickel, fettige, unreine und entzündliche Haut}

Bei Akne ist es wichtig, die Haut regelmäßig zu reinigen und von überschüssigem Talg und Hautfett zu befreien. Für eine entfettende/reinigende Wirkung von Heilerde reicht ein gut messerrückendicker ( $2-3 \mathrm{~mm}$ ) Auftrag von anfangs nicht zu festem Heilerdebrei (so fest wie Schlagsahne) oder der gebrauchsfertigen Paste, der lauwarm angelegt, in trockener warmer Luft nach rund einer halben Stunde durchtrocknet. Vor dem Abwaschen kann man einen Großteil der Schicht abbröseln. Dieses Abbröseln hat eine PeelingWirkung, bei der die oberflächliche Zellschicht der Außenhaut mitsamt den lose anliegenden Hautschuppen entfernt wird.

Man kann aber auch Heilerde mit einem fetten Öl wie Nachtkerzen- oder Jojobaöl mischen - dann trocknet die Maske nicht komplett aus. Wenn die Haut nach der Heilerde-Anwendung unangenehm trocken wird, schuppt und zum Hautjucken neigt, ist eine Nachfettung mit geeigneten Hautölen sinnvoll.

\section{Prospektive „Fernstudie“ bei unreiner Haut und Akne}

Im Rahmen einer prospektiven „Fernstudie“ untersuchte die Abteilung Naturheilkunde der Berliner Charité, inwieweit die kurmäßige Anwendung einer Heilerde-Gesichtsmaske das subjektiv empfundene Hauterscheinungsbild bei unreiner Haut und Akne beeinflusst. Insgesamt erhielten 250 Personen ein Paket mit Produkten, einer ausführlichen Anleitung, Fragebogen und Tagebuch. Angesichts eines Rürklaufs von 175 Fragebögen können die Ergebnisse als repräsentativ eingestuft werden.

Bis auf 2 Männer waren alle Teilnehmer weiblich und durchschnittlich 32 Jahre alt, der Anteil der Nichtraucher lag bei $70 \%$. Unreine Haut wurde von den Patienten seit durchschnittlich 16 Jahren beobachtet, hauptsächlich ausgelöst durch psychischen Stress (137 Nennungen), gefolgt von Schokolade (63 Nennungen); Sonnenbestrahlung, Östrogene (Pille) und Chemikalien wurden selten angeführt (jeweils weniger als 23 Nennungen). Die meisten Teilnehmer führten Art und Anzahl der Hauterscheinungen gewissenhaft auf. Der Ausgangswert der Effloreszenzen legt nahe, dass viele Teilnehmer durchaus mit der Diagnose Acne vulgaris zu versehen gewesen wären. Gemäß der Empfehlung wurden die Masken 2- bis 3-mal pro Woche angewendet (Durchschnittswert 2,5-mal für $16 \mathrm{Min}$.). Schwierigkeiten bei der Anwendung hatten nur $12 \%$ der Teilnehmer. Knapp zwei Drittel (65\%) spülten die Maske mit Wasser ab, 29\% rubbelten die angetrocknete Maske ab, um einen zusätzlichen Peeling-Effekt zu erzielen. 
- Tab. 3 Subjektiv beurteilte Abnahme von Hauteffloreszenzen in einer Anwendungsbeobachtung bei Probanden mit „unreiner“ Haut und Akne.

\begin{tabular}{|l|l|l|}
\hline $\begin{array}{l}\text { Art der } \\
\text { Effloreszenz }\end{array}$ & $\begin{array}{l}\text { Prozentualer } \\
\text { Rückgang }\end{array}$ & Veränderung gegenüber Ausgangswert \\
\hline Papeln & $-69 \%$ & $\begin{array}{l}\text { von durchschnittlich 5,5 auf 1,7 pro } \\
\text { Teilnehmer }\end{array}$ \\
\hline Kleine Zysten & $-58 \%$ & $\begin{array}{l}\text { von durchschnittlich 0,6 auf 0,25 pro } \\
\text { Teilnehmer }\end{array}$ \\
\hline Pickel & $-49 \%$ & $\begin{array}{l}\text { von durchschnittlich 9,3 auf 4,7 pro } \\
\text { Teilnehmer }\end{array}$ \\
\hline Mitesser & $-36 \%$ & $\begin{array}{l}\text { von durchschnittlich 34,3 auf 22,1 pro } \\
\text { Teilnehmer }\end{array}$ \\
\hline
\end{tabular}

Die Ergebnisse der 6-wöchigen Kur sind vor allem hinsichtlich ihrer deutlichen Ausprägung bemerkenswert ( A Abb. 5, \ Tab. 3). Bei der Anwendung von Heilerde wurden keine Nebenwirkungen beobachtet.

Das Hautgefühl wurde während der Anwendung mit der Schulnote 2,3 beurteilt, danach mit 2,0. Gut ein Viertel der Teilnehmer (28\%) entschied sich für eine Nachfettung nach der Heilerde-Maske. Viele Teilnehmer, die ihren Hautzustand ursprünglich als mäßig oder schlecht eingestuft hatten, beurteilten ihn am Ende der Anwendung als gut oder sehr gut - die Veränderung betrug durchschnittlich 0,78 -, also knapp eine Schulnote. Hautsymptome und Lebensqualität besserten sich deutlich. Entsprechend positiv fiel das Gesamturteil der Teilnehmer aus: Rund $80 \%$ der Teilnehmer bewerteten Empfindung, Hautgefühl und Wirksamkeit der Heilerde-Maske als gut oder sehr gut.

\section{Neurodermitis und Psoriasis}

Bei Neurodermitis und Schuppenflechte wird ein sanft kühlender Umschlag mit Luvos-Heilerde angelegt. Therapieziel ist die Linderung des Juckreizes und eine schonende Ablösung der Schuppen. Luvos-Heilerde hemmt zusätzlich die Sekretbildung und Blutung unter den abgelösten Schuppen und fördert die normale Wundheilung. LuvosHeilerde eignet sich nicht nur für die Behandlung von bereits vorhandenen schuppigen Herden, sondern auch zum Abmildern eines neuen Schubes, wenn man frühzeitig die entsprechenden Hautpartien damit behandelt.

\section{Cellulite}

Bei Cellulite entschlacken und straffen Körperwickel mit Luvos-Heilerde das Gewebe. Dazu wird ein Wickel ca. 20-30 Minuten an die betroffenen Stellen angelegt. Anschließend die Heilerde mit einem Tuch abfrottieren und Reste mit klarem Wasser abspülen. Der Wickel steigert die Durchblutung an den betroffenen Stellen und aktiviert den Stoffwechsel. Wickel werden regelmäßig 2- bis 3-mal pro Woche angewendet.

\section{Reduktion nach 6-wöchiger Anwendung}

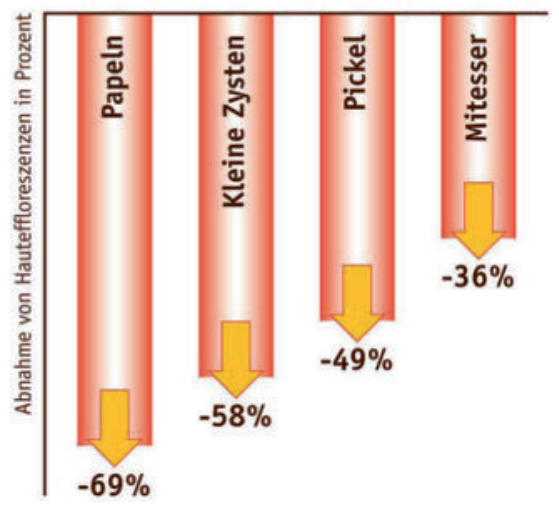

Abb. 5 Ergebnisse einer 6-wöchigen Anwendungsbeobachtung bei 175 Probanden mit „unreiner“ Haut oder Akne; subjektive Beurteilung.

\section{Anwendung bei Entzündungen}

Heilerde wird direkt auf Wunden und entzündete Stellen als salbenartige Paste aufgetragen. Hierzu gehören u. a. Verbrennungen, Sonnenbrand, Insektenstiche, oberflächliche Venenentzündungen (z. B. Krampfadern) und Entzündungen der Lymphgefäße.

Auf nässende Wunden bzw. Ulzera wird direkt das Pulver aufgestreut - es bildet sich zusammen mit den Wundsekreten eine Schutzschicht, unter der die Haut heilen kann.

Liegen Stauungszustände, z. B. im Rahmen von Krampfaderleiden (chronisch-venöse Insuffizienz) oder gar Lymphstauungen vor, ist es sinnvoll, die Heilerde dicker aufzutragen; diese schrumpft dann beim Trocknen und übt dadurch eine abflussfördernde Wirkung auf das Lymphsystem und die Venen aus, wie man sie sonst durch Kompressionsmaßnahmen oder Lymphdrainage erreicht. Hierzu wird ein zähflüssiger Heilerde-Brei mindestens 5 mm dick aufgetragen und ein nichtelastisches Gewebe als Verstärkung herumgewickelt.

\section{Anwendung bei Muskel- und Gelenkbeschwerden}

Ein weiteres Anwendungsgebiet sind rheumatische Beschwerden und verletzungsbedingte Entzündungen. Auflagen mit feuchtem Heilerde-Brei beeinflussen wie andere Kneipp-Anwendungen die Temperaturregulation im betroffenen Hautgebiet.

Kalte Anwendungen sind das Mittel der Wahl bei akutentzündlichen Beschwerden, da Kälte das Schmerzempfinden verringert und so zur Linderung der Beschwerden beiträgt. Die Kühlwirkung kann intensiviert werden, indem der Heilerde-Brei vorher in den Kühlschrank gestellt und dann relativ flüssig und dennoch streichfähig sowie nicht zu dünn aufgebracht wird. 
Hauptanwendungsgebiete lokaler Kühlbehandlungen sind entzündete Gelenke im Rahmen von Verletzungen oder entzündlichen und degenerativen rheumatischen Beschwerden (Arthritis und Arthrosen). Kühlbehandlungen sind darüber hinaus bei Venenentzündungen und bei venösen Stauungen (schwere Beine) angezeigt. Weiterhin können reflektorische Wirkungen auf innere Organe genutzt werden und die allgemein harmonisierende Wirkung einer Auflagentherapie, wenn der Wickel nicht zu kalt angelegt wird und die allmähliche Erwärmung erlebt wird. In diesem Punkt unterscheidet sich Heilerde-Brei nur geringfügig von anderen Peloiden wie Fango, Moor usw. und könnte auch warm-heiß angewendet werden.

Warme Anwendungen werden hauptsächlich bei schleichend degenerativen Beschwerden eingesetzt, da sie für eine bessere Durchblutung der Muskulatur und damit für eine wohltuende Entkrampfung sorgen. Bei warmen Anwendungen wird die Paste dickflüssiger angerührt. Grundsätzlich gilt: Je größer die zu behandelnde Fläche ist, desto dünner sollte die Heilerde-Paste aufgetragen werden.

\section{KERNAUSSAGEN}

Heilerde ist ein bewährtes Naturheilmittel, das in neueren Studien seinen Nutzen bei Refluxkrankheit, Reizmagen und sogar bei Reizdarm zeigen konnte. Besonders interessant ist seine entgiftende Wirkung bei Histaminintoleranz und beim Fasten. Aber auch äußerlich eignet sich Heilerde bei Hauterkrankungen, z. B. bei Entzündungen, Akne oder Neurodermitis und Psoriasis. Sie findet in Form von kalten oder warmen Pasten zudem bei Muskel- und Gelenkbeschwerden Anwendung.

\section{Interessenkonflikt}

Prof. Bernhard Uehleke berät die Heilerde-Gesellschaft Luvos Just GmbH \& Co. KG und hält Vorträge über Heilerde. Er berät weitere Anbieter von meist pflanzlichen Arzneimitteln, Medizinprodukten und Nahrungsergänzungsmitteln.

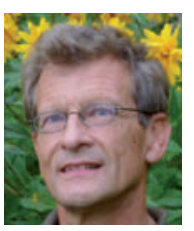

Prof. Dr. med. Dr. rer. nat. Bernhard Uehleke

Prof. Dr. med. Dr. rer. nat. Bernhard Uehleke arbeitete in leitenden Funktionen in der Pharmaindustrie, bevor er sich vor 30 Jahren der Naturheilkunde zuwandte. Er baute in Bad Wörishofen ein Forschungsinstitut auf, wechselte später an universitäre Abteilungen für Naturheilkunde, Klinische Pharmakologie und Phytotherapie. Seine Hauptforschungsgebiete als Forschungsleiter der Abt. für klinische Naturheilkunde der Charité Berlin sind Heilpflanzen, Klostermedizin, Kneipptherapie und gesunde Ernährung bzw. Heilfasten.

\section{Korrespondenzadresse}

\section{Prof. Dr. med. Dr. rer. nat. Bernhard Uehleke}

Abt. Klinische Naturheilkunde der Charité Universitätsmedizin Berlin

im Immanuel-Krankenhaus Wannsee

Königstr. 63

14109 Berlin-Wannsee

E-Mail: b.uehleke@immanuel.de

\section{Literatur}

[1] Graner und Partner GmbH. Bestimmung der mobilisierbaren Inhaltsstoffe in Prozent. Untersuchungsbericht vom 11.11.2009

[2] Kompendium Gastroenterologie 2012; 8 (1)

[3] Kompendium Gastroenterologie 2011; 7 (1)

Bibliografie

DOI https://doi.org/10.1055/a-0979-0257

Ernährung \& Medizin 2019; 34: 199-207

(c) Georg Thieme Verlag KG Stuttgart · New York

ISSN 1439-1635 